\title{
Aparecimento de folhas em mudas de eucalipto estimado por dois modelos
}

\author{
Fabrina Bolzan Martins ${ }^{(1)}$ e Nereu Augusto Streck(1)
}

\author{
(1)Universidade Federal de Santa Maria, Centro de Ciências Rurais, Dep. de Fitotecnia, CEP 97105-900 Santa Maria, RS. \\ E-mail: fabrinab@hotmail.com, nstreck2@yahoo.com.br
}

\begin{abstract}
Resumo - O objetivo deste trabalho foi avaliar e comparar os modelos do Filocrono e de Wang e Engel para estimativa do aparecimento de folhas em mudas de Eucalyptus grandis e E. saligna. Foram instalados dois experimentos em Santa Maria em 2005 e 2006, um, em campo, com nove épocas de semeadura, e o outro, em casa, de vegetação com duas épocas de semeadura. Os modelos usados foram o do Filocrono, que assume uma relação linear entre taxa de aparecimento de folhas e temperatura, e o de Wang e Engel, que assume uma relação não-linear entre taxa de aparecimento de folhas e temperatura. As quatro primeiras épocas de semeadura em campo foram usadas para estimar os coeficientes dos modelos utilizados. As épocas de semeadura restantes do experimento em campo e as duas épocas de semeadura, em casa de vegetação, foram utilizadas como dados independentes para avaliar os modelos. O modelo de Wang e Engel proporcionou estimativa mais precisa do número de folhas, com valor da raiz do quadrado médio do erro de 2,7 e 3,7 folhas, comparado com o modelo do Filocrono com 7,1 e 10 folhas para E. grandis e E. saligna, respectivamente.
\end{abstract}

Termos para indexação: Eucalyptus grandis, Eucalyptus saligna, desenvolvimento vegetal, soma térmica, taxa de aparecimento de folhas.

\section{Leaf appearance in seedlings of eucalyptus estimated by two models}

\begin{abstract}
The objective of this work was to evaluate and compare the Phyllochron and the Wang and Engel models for estimation of leaves appearance in seedlings of Eucalyptus grandis and E. saligna. Two experiments were carried out in Santa Maria during 2005 and 2006. One was a field experiment with nine sowing dates, and the other was a greenhouse experiment with two sowing dates. Phyllochron model assumes a linear relationship between leaf appearance rate and temperature, and the Wang and Engel model assumes a non-linear relationship between leaf appearance number and temperature. Four sowing dates from the field experiment were used to estimate the models coefficients and the other sowing dates of the field and greenhouse experiments were used as independent data to evaluate the models. The Wang and Engel model better estimated the number of leaves, comprising a root mean square error (RMSE) of 2.7 and 3.7 leaves compared with the Phyllocron model with RMSE of 7.1 and 10 leaves for E. grandis and E. saligna, respectively.
\end{abstract}

Index terms: Eucalyptus grandis, Eucalyptus saligna, plant development, thermal time, leaf appearance rate.

\section{Introdução}

O gênero da família Myrtaceae e subfamília Leptospermoideae mais valorizado comercialmente é o Eucalyptus, o qual foi introduzido em praticamente todos os continentes (Stape et al., 2004). É cultivado em larga escala por suas características favoráveis como rápido crescimento, facilidade de manejo, diversidade de espécies e atendimento a vários propósitos industriais (Santos et al., 2003).

Espécies do gênero Eucalyptus compõem $40 \%$ das plantas arbóreas introduzidas nos países tropicais
(Kallarackal \& Somen, 1997). Esse é o gênero florestal predominante e mais produtivo do Brasil, com incremento médio anual de 9 a $39 \mathrm{Mg} \mathrm{ha}^{-1} \mathrm{ano}^{-1} \mathrm{em}$ aproximadamente 3 milhões de hectares plantados e manejados intensivamente principalmente para a obtenção dos produtos como polpa celulósica, papel, madeira para serraria e geração de energia (Associação Brasileira de Florestas Plantadas, 2006). O Rio Grande do Sul é o quinto maior produtor de eucalipto do Brasil com uma área plantada de 179.690 ha, dos quais aproximadamente $30 \%$ são plantados com Eucalyptus grandis e 10\% com Eucalyptus saligna (Associação Brasileira de Florestas Plantadas, 2006). Observa-se 
aumento na área plantada de eucalipto nas regiões tropicais, atribuído à ênfase em pesquisas com modelagem ecofisiológica (Stape et al., 2004).

O desenvolvimento das plantas é afetado por fatores bióticos e abióticos (Streck, 2002). Entre os fatores abióticos, a temperatura do ar é o fator que mais influencia o desenvolvimento das plantas (Hodges, 1991) incluindo o eucalipto (Scurfield, 1961). O número de folhas acumuladas na haste principal (NF) é uma excelente medida de desenvolvimento vegetal (Hodges, 1991) e está associada à evolução da área foliar da planta, a qual determina a interceptação da radiação solar usada na fotossíntese do dossel vegetativo. Portanto, a estimativa do NF é de interesse em estudos ecofisiológicos das culturas agrícolas e florestais. O NF pode ser estimado por meio da taxa de aparecimento de folhas (TAF). Ao se integrar a TAF, o resultado é o NF (Wang \& Engel, 1998). Uma das maneiras de estimar a TAF é por meio do conceito de Filocrono, definido como o intervalo de tempo para o aparecimento de folhas sucessivas na haste principal (Klepper et al., 1982; Xue et al., 2004). Outra forma de estimar a TAF é através de modelos multiplicativos compostos por funções de resposta que descrevem o efeito dos fatores ambientais sobre a emissão de folhas, como por exemplo, o modelo de Wang e Engel (Wang \& Engel, 1998).

No modelo do Filocrono pressupõe-se uma relação linear entre TAF e temperatura do ar (Xue et al., 2004). Com o tempo expresso em soma térmica (ST) com unidade ${ }^{\circ} \mathrm{C}$ dia, o Filocrono tem como unidade ${ }^{\circ} \mathrm{C}$ dia por folha (Streck et al., 2006). O modelo do Filocrono é utilizado para estimar o desenvolvimento na maioria das culturas (Streck, 2002; Xue et al., 2004), mas apresenta desvantagens, como a suposição de que a resposta da taxa de desenvolvimento é linearmente proporcional à temperatura (Yan \& Hunt, 1999). Processos biológicos, incluindo a TAF, têm resposta não-linear à temperatura (Streck, 2002; Xue et al., 2004).

No modelo de Wang e Engel (WE), a função de resposta da TAF à temperatura do ar [f(T)] é não-linear e descrita por uma função beta (Wang \& Engel, 1998). A $\mathrm{f}(\mathrm{T})$ é multiplicada pela taxa de aparecimento de folhas máxima e por meio dessa multiplicação calcula-se a taxa atual de aparecimento das folhas (Xue et al., 2004).

Estudos demonstraram que o modelo de WE oferece melhor estimativa do NF, em culturas agrícolas anuais, do que o modelo do Filocrono, porém este último continua sendo utilizado amplamente na estimativa do desenvolvimento das culturas (Xue et al., 2004).
Em espécies agrícolas, estudos que relacionam parâmetros de desenvolvimento com temperatura do ar são freqüentes, enquanto em espécies florestais, não há ênfase nesse tipo de estudo (Sands \& Landsberg, 2002).

O objetivo deste trabalho foi avaliar e comparar os modelos do Filocrono e de Wang e Engel para estimativa do aparecimento de folhas em mudas de E. grandis e E. saligna.

\section{Material e Métodos}

Foram conduzidos dois experimentos no Dep. de Fitotecnia da Universidade Federal de Santa Maria (Santa Maria, RS, 29² $3^{\prime} \mathrm{S}, 5^{\circ} 43^{\prime} \mathrm{W}$ e $95 \mathrm{~m}$ de altitude). O primeiro experimento foi conduzido em campo, em nove épocas de semeadura e o segundo experimento foi conduzido em casa de vegetação em duas épocas de semeadura, a fim de se obter mudas de eucalipto desenvolvidas em condições variadas de temperatura. Foram usadas duas espécies de eucalipto, E. grandis e E. saligna, cujas sementes foram adquiridas da Estação Experimental de Silvicultura da Fundação Estadual de Pesquisa Agropecuária (Fepagro), localizada no distrito de Boca do Monte, em Santa Maria, RS.

No experimento de campo, foi utilizado o delineamento em blocos ao acaso, em esquema fatorial (Fator A: espécie, Fator D: épocas de semeadura) com três repetições, nove épocas de semeadura e duas espécies. Cada parcela foi constituída de dois vasos de plástico, cor preta, com capacidade de $12 \mathrm{~L}$ por espécie. Os vasos foram preenchidos com Argissolo Vermelho-Amarelo distrófico típico, do horizonte $\mathrm{B}$ pertencente à unidade de mapeamento São Pedro (Embrapa, 1999), e enterrados a fim de minimizar o aquecimento do solo pela incidência direta de radiação solar nas paredes externas dos vasos, o que poderia influenciar a taxa de aparecimento de folhas. O espaçamento entre vasos foi de $50 \mathrm{~cm}$.

A semeadura da época 1 (CA1) foi em 25/5/2005 e a data da emergência, considerada quando $50 \%$ das plantas estavam visíveis acima do solo, ocorreu em 8/6/2005 em ambas as espécies. Na época 2 (CA2) a semeadura foi em 20/6/2005 e a emergência ocorreu em 1/7/2005 em ambas as espécies. Na época 3 (CA3) a semeadura foi em 15/7/2005 com emergência em 1/8/2005 em ambas as espécies. Na época 4 (CA4) a semeadura foi em 22/9/2005 e a emergência ocorreu em 29/9/2005 e 30/9/2005 em E. grandis e E. saligna, respectivamente. Na época 5 (CA5), a semeadura foi em 20/10/2005 com emergência em 25/10/2005 para ambas espécies. 
Na época 6 (CA6), a semeadura ocorreu em 21/11/2005 e a emergência, em 26/11/2005 e 27/11/2005 para E. grandis e E. saligna, respectivamente. Na época 7 (CA7), a semeadura foi em 13/12/2005 e a emergência, em 19/12/2005 e 20/12/2005 para E. grandis e E. saligna, respectivamente. $\mathrm{Na}$ época 8 e na época 9 , foram utilizadas sementes pré-germinadas em laboratório a $25^{\circ} \mathrm{C}$ por quatro dias, pois houve dificuldade na germinação e no estabelecimento das plântulas no campo em virtude de altas temperaturas e de chuvas intensas. Nessas duas épocas, a data de emergência foi considerada a mesma data de transplante que, na época 8 (CA8), foi em 14/2/2006 e, na época 9 (CA9), em 3/4/2006 em ambas espécies.

Os dados de temperatura mínima e máxima do ar em campo foram obtidos em uma estação meteorológica convencional, pertencente ao 8o Distrito de Meteorologia, do Instituto Nacional de Meteorologia, localizada a, aproximadamente, $150 \mathrm{~m}$ da área experimental.

No experimento em casa de vegetação, o delineamento experimental utilizado foi o inteiramente casualizado, com 14 repetições por espécie em duas épocas de semeadura. As semeaduras foram em 3/10/2005 (época 1 - CV1) e 12/5/2006 (época 2 - CV2) e a emergência ocorreu em 11/10/2005 (época 1) e 30/5/2006 (época 2), respectivamente. Neste experimento, cada repetição foi constituída de um vaso de plástico, cor preta, com capacidade de $8 \mathrm{~L}$ preenchido com o mesmo substrato do experimento em campo. Os vasos foram colocados em uma bancada de concreto de $70 \mathrm{~cm}$ de altura e um espaçamento de $20 \mathrm{~cm}$ entre vasos. Todos os vasos foram revestidos com papel-jornal a fim de minimizar a incidência de radiação solar, e evitar a elevação da temperatura do substrato. As sementes utilizadas no experimento de casa de vegetação foram obtidas do mesmo lote do experimento em campo.

As temperaturas mínima e máxima diárias do ar, no interior da casa de vegetação, foram medidas com um termômetro de mínima de álcool e de um termômetro de máxima de mercúrio, instalados dentro de um miniabrigo meteorológico de madeira pintado de branco e instalado na altura e ao lado da bancada onde as plantas localizavam-se.

A correção da acidez e nutrientes do substrato usado nos dois experimentos foi realizada um mês antes de cada semeadura, conforme análise de solo e recomendação técnica para as espécies (Sociedade Brasileira de Ciência do Solo, 2004). Os vasos foram irrigados durante o período experimental a fim de evitar deficiência hídrica nos dois experimentos.
Uma semana após a emergência, duas plantas por vaso foram selecionadas ao acaso e marcadas com arames coloridos. Nos dois experimentos foram realizados dois desbastes nas outras plantas, o primeiro quando as plantas tinham duas folhas visíveis na haste principal, e o segundo, quando tinham quatro folhas visíveis, restando apenas duas plantas marcadas por vaso. As brotações axilares foram removidas à medida que foram aparecendo nas plantas, mantendo-se apenas a haste principal em crescimento.

O número de folhas emitidas ou acumuladas na haste principal (NF) das plantas marcadas foi contado uma vez por semana, no experimento de campo, e a cada três dias, no experimento na casa de vegetação. As folhas foram consideradas visíveis quando apresentaram comprimento do limbo de no mínimo $1 \mathrm{~cm}$. A contagem iniciou-se a partir de duas folhas visíveis e terminou quando o valor de NF foi aproximadamente 25 , quando se considerou que a planta não mais se encontrava na fase de muda (Carneiro, 1995).

Na estimativa do aparecimento de folhas, foram usados os modelos de Filocrono (Matthews \& Hunt, 1994; Xue et al., 2004) e o modelo de WE (Wang \& Engel, 1998). Em ambos os modelos, são necessárias as temperaturas cardinais das duas espécies de eucalipto. As temperaturas cardinais de E. saligna foram $8^{\circ} \mathrm{C}$ para a temperatura base ou mínima (Tb) (Martins, 2007), $25^{\circ} \mathrm{C}$ para a temperatura ótima (Tot) (Martins, 2007) e $35^{\circ} \mathrm{C}$ para a temperatura máxima (Tmáx) (Nieto \& Rodriguez, 2003). As temperaturas cardinais de E. grandis foram $\mathrm{Tb}=10^{\circ} \mathrm{C}$ (Martins, 2007), Tot $=27^{\circ} \mathrm{C}($ Martins, 2007) $\mathrm{e}$ Tmáx $=36^{\circ} \mathrm{C}$ (Almeida et al., 2004).

No modelo do Filocrono, foi calculada a regressão linear entre número de folhas acumuladas na haste principal (NF) e soma térmica acumulada (STa) a partir da data de emergência, pela seguinte fórmula geral (Frank $\&$ Bauer, 1995):

$\mathrm{NF}=\mathrm{a} \times \mathrm{STa}+\mathrm{b}$

em que a e b são os coeficientes angular e linear da regressão linear e STa $\left({ }^{\circ} \mathrm{C}\right.$ dia $)$ é calculada por STa $=\Sigma \mathrm{STd}$, sendo STd a soma térmica diária $\left({ }^{\circ} \mathrm{C}\right.$ dia) a partir da data de emergência, calculada em função das três temperaturas cardinais (Matthews \& Hunt, 1994; Streck, 2002) pela equação:

$\mathrm{STd}=(\mathrm{T} \overline{\mathrm{m}}-\mathrm{Tb}) \times 1 \mathrm{dia}$

quando $\mathrm{Tb}<\mathrm{T} \overline{\mathrm{m}} \leq \mathrm{Tot}$ e se $\mathrm{T} \overline{\mathrm{m}}<\mathrm{Tb}$, então $\mathrm{T} \overline{\mathrm{m}}=\mathrm{Tb}$ e por $\mathrm{STd}=($ Tot $-\mathrm{Tb})[(\mathrm{T} \bar{m}-\mathrm{Tmáx}) /($ Tot - Tmáx $)] \times 1$ dia (3) quando Tot $<\mathrm{T} \overline{\mathrm{m}} \leq$ Tmáx e se $\mathrm{T} \overline{\mathrm{m}}>$ Tmáx então $\mathrm{T} \overline{\mathrm{m}}=$ Tmáx, sendo

$\mathrm{T} \overline{\mathrm{m}}=(\mathrm{TM}+\mathrm{Tm}) / 2$ 
em que Tot é a temperatura ótima da espécie $\left({ }^{\circ} \mathrm{C}\right)$, Tb é a temperatura base ou mínima da espécie $\left({ }^{\circ} \mathrm{C}\right)$, Tmé a temperatura média diária do ar $\left({ }^{\circ} \mathrm{C}\right)$, Tmáx é a temperatura máxima da espécie $\left({ }^{\circ} \mathrm{C}\right), \mathrm{TM}$ é a temperatura máxima diária do ar $\left({ }^{\circ} \mathrm{C}\right)$, Tm é a temperatura mínima diária do $\operatorname{ar}\left({ }^{\circ} \mathrm{C}\right)$.

O Filocrono $\left({ }^{\circ} \mathrm{C}\right.$ dia por folha) foi calculado tomando-se o inverso do coeficiente angular da regressão linear (equação 1), ou seja, Filocrono = 1/a (Frank \& Bauer, 1995; Xue et al., 2004; Streck et al., 2006). O número de folhas acumuladas na haste principal (NF) foi calculado por: $\mathrm{NF}=\mathrm{STa}$ /Filocrono (Streck et al., 2006).

O modelo de WE (Wang \& Engel, 1998) utiliza uma função de resposta não-linear de temperatura [f(T)], combinada com um fator multiplicativo $\left(\mathrm{TAF}_{\text {máx }}\right)$. $\mathrm{O} \mathrm{TAF}_{\text {máx }}$ é a taxa máxima de aparecimento de folhas (folhas por dia), que é a taxa de aparecimento de folhas quando a temperatura do ar é igual a temperatura ótima da espécie. O modelo de WE tem a fórmula geral:

$\mathrm{TAF}=\mathrm{TAF}_{\text {máx }} \times \mathrm{f}(\mathrm{T})$

em que TAF é a taxa de aparecimento de folhas (folhas por dia).

A $\mathrm{f}(\mathrm{T})$ no modelo de WE é uma função beta definida como:

$\mathrm{f}(\mathrm{T})=\left[2(\mathrm{~T} \overline{\mathrm{m}}-\mathrm{Tb})^{\alpha}(\mathrm{Tot}-\mathrm{Tb})-(\mathrm{T} \overline{\mathrm{m}}-\mathrm{Tb})^{2 \alpha}\right] /(\mathrm{Tot}-\mathrm{Tb})^{2 \alpha}(6)$ quando $\mathrm{Tb}<\mathrm{T} \overline{\mathrm{m}} \leq \mathrm{Tmáx}$, e $\mathrm{f}(\mathrm{T})=0$ quando $\mathrm{T} \overline{\mathrm{m}}<\mathrm{Tb}$ ou T⿳亠m⿲丿八 Tmáx.

em que Tb, Tot e Tmáx são as temperaturas cardinais, mínima, ótima e máxima, de cada espécie $\left({ }^{\circ} \mathrm{C}\right), \mathrm{T} \bar{m}$ é a temperatura média diária do ar $\left({ }^{\circ} \mathrm{C}\right)$.

$\mathrm{O}$ coeficiente $\alpha$ é calculado por:

$\alpha=\ln (2) / \ln [(\mathrm{Tmáx}-\mathrm{Tb}) /(\mathrm{Tot}-\mathrm{Tb})]$

em que ln é o logaritmo natural.

Para calcular o número de folhas (NF) no modelo de WE, somam-se os valores diários de TAF, ou seja, $\mathrm{NF}=\Sigma \mathrm{TAF}$, a partir da data de emergência (Wang \& Engel, 1998; Streck et al., 2003).

Os coeficientes a do modelo do Filocrono (equação 1)

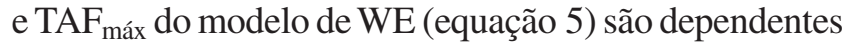
da espécie e foram estimados usando-se os dados das épocas de semeadura 1, 2, 3 e 4 do experimento em campo. Na determinação do coeficiente a do modelo do Filocrono, foi realizada a média aritmética dos coeficientes angulares da equação 1 estimados nas épocas $1,2,3$ e 4 . O valor do coeficiente a para E. grandis foi de 0,03239 (Filocrono $=30,9^{\circ} \mathrm{C}$ dia por folha) e para E. saligna foi de 0,03101 (Filocrono $=32,2^{\circ} \mathrm{C}$ dia por folha). Na determinação do coeficiente $\mathrm{TAF}_{\text {máx }}$ do modelo de $\mathrm{WE}$, foram estimadas equações de regressão não-linear múltipla para os valores do NF acumulado em função dos valores acumulados de $\mathrm{f}(\mathrm{T})$. A equação de regressão forneceu o valor do coeficiente $\mathrm{TAF}_{\text {máx }}$ baseado no menor valor do quadrado médio do erro (QME) (Streck et al., 2006, 2007). O valor do coeficiente $\mathrm{TAF}_{\text {máx }}$ para E. grandis foi de 0,4107 folhas por dia e para E. saligna foi de 0,3180 folhas por dia.

Para avaliar a performance dos modelos, foram utilizados os valores de NF estimados pelos modelos e os valores de NF observados nas épocas 5, 6, 7, 8, 9 do experimento em campo e, nas duas épocas do experimento, em casa de vegetação. A homogeneidade da variância entre valores de NF observados e estimados pelos dois modelos, em cada época, nas duas espécies, foi testada com o teste de Bartlett, em que a hipótese H0 (as variâncias são homogêneas) foi testada contra a hipótese H1 (as variâncias não são homogêneas). O mesmo teste de Bartlett pode ser usado para testar a ausência de normalidade das amostras, o que também foi feito neste estudo. As médias de NF observadas foram comparadas com as médias de NF estimadas pelos dois modelos em cada época nas duas espécies com o teste $t$. Os desvios da estimativa de NF pelos dois modelos, em cada época, nas duas espécies, foram testados, assumindo-se que o quadrado médio do erro (QME), calculado por $\Sigma(\mathrm{Ei}-\mathrm{Oi})^{2} / \mathrm{n}$, em que Ei são os valores estimados; Oi são os valores observados; e n é o número de observações, dos dois modelos seguem a distribuição de F, ou seja, $\left.\left(\mathrm{QME}_{\text {Filocrono }} / \mathrm{QME} \mathrm{EE}_{\mathrm{WE}}\right) \cap \mathrm{F}\right)$.

Outras estatísticas empíricas utilizadas para avaliar a performance dos modelos foram o coeficiente de correlação (r) (Camargo \& Sentelhas, 1997), o índice dw de Willmott (Willmott, 1981), o índice c (Camargo \& Sentelhas, 1997), a raiz do quadrado médio do erro (RQME) (Streck, 2002; Xue et al., 2004) e BIAS (Leite $\&$ Andrade, 2002).

A estatística r permite quantificar o grau de associação entre duas ou mais variáveis (Schneider, 1998). O valor de $\mathrm{r}$ varia de -1 a 1 e quanto maior o valor absoluto de $\mathrm{r}$ (seja positivo ou negativo) maior a adesão entre os valores observados e os valores estimados. $\mathrm{O}$ valor de $\mathrm{r}$ é dado pela fórmula geral (Schneider, 1998):

$\mathrm{r}=\sum_{\mathrm{i}=1}^{\mathrm{n}}(\mathrm{Oi}-\overline{\mathrm{O}})(\mathrm{Ei}-\overline{\mathrm{E}}) /\left\{\left[\sum_{i=1}^{\mathrm{n}}(\mathrm{Oi}-\overline{\mathrm{O}})^{2}\right]\left[\sum_{i=1}^{\mathrm{n}}(\mathrm{Ei}-\overline{\mathrm{E}})^{2}\right]\right\}^{0,5}$

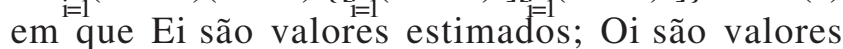
observados; $\overline{\mathrm{E}}$ é a média dos valores estimados; Ōé a média dos valores observados.

O índice de concordância, designado dw, proporciona a exatidão do modelo. Seus valores variam de zero, para 
nenhuma concordância, a 1, para a concordância perfeita (Willmott, 1981). O valor de dw é calculado pela fórmula (Camargo \& Sentelhas, 1997):

$\mathrm{dw}=1-\left\{\sum_{i=1}^{\mathrm{n}}(\mathrm{Ei}-\mathrm{Oi})^{2} /\left[\sum_{i=1}^{\mathrm{n}}(|\mathrm{Ei}-\overline{\mathrm{O}} \mathrm{l}+| \mathrm{Oi}-\overline{\mathrm{O}} \mid)^{2}\right]\right\}$

em que E1 são valores estimados; Oi são valores observados; Ō é a média dos valores observados.

$\mathrm{O}$ índice c fornece o desempenho do modelo, e é classificado pela Tabela 1 e obtido pela fórmula (Camargo \& Sentelhas, 1997):

$\mathrm{C}=\mathrm{r} \times \mathrm{dw}$

em que r é o coeficiente de correlação; dw é o índice de concordância.

O RQME expressa o erro produzido pelo modelo, quanto menor o valor dessa estatística, melhor o modelo. O valor do RQME é calculado pela fórmula (Streck, 2002):

$\mathrm{RQME}=\left[\sum_{i=1}^{\mathrm{n}}(\mathrm{Ei}-\mathrm{Oi})^{2} / \mathrm{n}\right]^{0,5}$

em que Ei são valores estimados; Oi são valores observados; n é o número de observações.

O índice BIAS expressa a tendência do modelo, sendo o desvio médio dos valores estimados em relação aos observados (Schneider, 1998). Quanto mais próximo de zero for o valor dessa estatística, menor é a tendência do modelo e melhor é o modelo. O valor do BIAS é dado pela fórmula geral (Leite \& Andrade, 2002):

$\mathrm{BIAS}=\left(\sum_{i=1}^{\mathrm{n}} \mathrm{Ei}-\sum_{\mathrm{i}=1}^{\mathrm{n}} \mathrm{Oi}\right) / \sum_{\mathrm{i}=1}^{\mathrm{n}} \mathrm{Oi}$

$\mathrm{Na}$ escolha do melhor modelo, foi adotada uma classificação usando-se o método dos valores ponderados dos escores estatísticos (Vp) (Thiersch, 1997). Na obtenção do Vp, é atribuído pesos de 1 a n aos indicadores estatísticos, sendo n o número total de modelos a serem avaliados. Como neste trabalho são dois modelos adotaram-se os valores 1 e 2 . Assim o valor 1 é atribuído ao melhor modelo e o valor 2 é atribuído ao pior modelo em cada indicador estatístico. O Vp foi calculado somando-se todos os pesos atribuídos a cada indicador estatístico em cada época de

Tabela 1. Critérios de interpretação do desempenho dos modelos pelo índice c (adaptado de Camargo \& Sentelhas, 1997).

\begin{tabular}{ll}
\hline Valor de c & Desempenho \\
\hline$>0,85$ & Ótimo \\
0,76 a 0,85 & Muito bom \\
0,66 a 0,75 & Bom \\
0,61 a 0,65 & Moderado \\
0,51 a 0,60 & Fraco \\
0,41 a 0,50 & Muito fraco \\
$\leq 0,40$ & Péssimo \\
\hline
\end{tabular}

semeadura. O melhor modelo foi o que apresentou o menor valor ponderado (Thiersch, 1997).

\section{Resultados e Discussão}

Durante as nove épocas de semeadura no campo houve variação na temperatura mínima e máxima do ar, assim como nas duas épocas de semeadura em casa de vegetação. No experimento de campo, o menor valor de temperatura mínima diária do ar foi de $-0,2^{\circ} \mathrm{C}$ observado na época de semeadura 9 (CA9) e o maior valor de temperatura máxima diária do ar foi de $38,6^{\circ} \mathrm{C}$ observado nas épocas de semeadura 6 (CA6) e 7 (CA7). No experimento em casa de vegetação, o menor valor de temperatura mínima diária do ar foi 9,4 e $2^{\circ} \mathrm{C}$ nas épocas 1 (CV1) e 2 (CV2), respectivamente, e o maior valor de temperatura máxima diária do ar foi 43,4 e $38,9^{\circ} \mathrm{C}$ nas épocas $\mathrm{CV} 1$ e CV2, respectivamente. Essas diferentes condições de temperatura do ar afetaram a velocidade de emissão de folhas na haste principal e são importantes em estudos de desenvolvimento e crescimento vegetal (Scurfield, 1961) e na calibração e avaliação de modelos matemáticos para a estimativa da TAF (Streck et al., 2003).

Nas épocas CA6 e CA7, quando as temperaturas mínima, média e máxima foram mais elevadas, a velocidade de emissão das folhas foi maior, e o contrário ocorreu com as épocas CA1, CA2 e CA9, quando as temperaturas foram baixas e, conseqüentemente, a velocidade de emissão das folhas foi baixa.

$\mathrm{O}$ teste de Bartlett indicou que os pressupostos da normalidade foram atendidos para os valores de NF observados e estimados pelos dois modelos. Ainda ao aplicar-se este teste, a hipótese H0 (as variâncias são homogêneas) não foi rejeitada em todas as épocas e nas duas espécies para ambos modelos (Tabela 2), indicando homogeneidade de variância entre valores de NF observados e estimados pelos dois modelos, o que é desejável, pois os dados são homocedásticos (variância constante). A média do NF estimada foi geralmente maior que a média do NF observada. Entre os modelos, a média do NF estimada foi sempre maior com o modelo do Filocrono comparado com o modelo de WE para as duas espécies (Tabela 2).

A média de NF estimada pelo modelo do Filocrono foi estatisticamente superior à média observada em todas as épocas para $E$. grandis e em cinco das sete épocas para $E$. saligna. Já a média de NF estimada pelo modelo de WE não diferiu estatisticamente da média observada 
em três das sete épocas para ambas espécies, e nas épocas em que diferiu estatisticamente da média observada, a diferença foi de no máximo cinco folhas, valor bem inferior ao valor máximo de 15 folhas obtido na pior estimativa com o modelo do Filocrono. O QME, em geral, foi estatisticamente menor com o modelo de WE, comparado com o modelo do Filocrono nas diferentes épocas em ambas espécies (Tabela 2). Apenas em duas épocas (CV1 e CV2, para E. grandis, e CA6 e CV1, para E. saligna) o QME foi maior com o modelo do filocrono, mas pelo teste $\mathrm{F}$ apenas os QME das duas épocas para E. saligna (CA6 e CV1) foram diferentes. Estes resultados indicam melhor desempenho do modelo de WE comparado com o modelo do Filocrono, e entre espécies, melhores estimativas de NF para o E. grandis do que para o E. saligna.

A análise visual dos dados observados e estimados também indica que o modelo de WE estimou melhor o NF que o modelo do Filocrono para E. grandis e E. saligna, com valores menores de RQME (Figura 1). Para ambas espécies, os valores estimados pelo método de WE estão mais próximos da linha 1:1 do que os valores estimados pelo modelo do Filocrono.

O valor do RQME, para o modelo do Filocrono, foi de 7,1 folhas para E. grandis e 10 folhas para
E. saligna, enquanto o valor do RQME, para o modelo de WE, foi de 2,7 folhas para E. grandis e 3,7 folhas para E. saligna (Figura 1). Um erro de três a quatro folhas pode ser considerado baixo e aceitável em mudas de eucalipto, uma vez que as folhas das duas espécies estudadas na fase de muda apareceram sempre aos pares, o que significa um erro de apenas um ou dois nós e, em dias do calendário civil, um erro de um ou dois nós representa um erro menor que uma semana, de três a seis dias (Mitchell, 1997).

$\mathrm{Na}$ análise das estatísticas empíricas (Tabela 3), confirma-se que para E. grandis o modelo de WE foi superior ao modelo do Filocrono em todas as épocas de semeadura a campo e também no experimento em casa de vegetação. Os valores dos índices estatísticos dw, r e c foram maiores no modelo de WE em todas as épocas, variando de 0,8675 a 0,9943 para o índice dw, 0,9892 a 0,9996, para o índice r, e 0,8595 a 0,9939, para o índice c e, segundo a Tabela 1, todos apresentam desempenho ótimo. Os índices RQME e BIAS apresentaram os menores valores para o modelo de WE e variaram entre 0,8572 a 5,0704 para RQME e entre 0,0237 a 0,2768 para o BIAS. Na análise dos valores de BIAS, os dois modelos geralmente superestimaram o NF em pequena amplitude de valores, exceto para a primeira época de semeadura

Tabela 2. Média, variância e quadrado médio do erro (QME) do número de folhas acumuladas na haste principal usadas na avaliação dos modelos do Filocrono (FIL) e Wang e Engel (WE) para estimativa do aparecimento de folhas em duas espécies de eucalipto, nas diferentes épocas de semeadura a campo (CA5, CA6, CA7, CA8, CA9) e em casa de vegetação (CV1 e CV2) ${ }^{(1)}$.

\begin{tabular}{|c|c|c|c|c|c|c|c|c|c|}
\hline \multirow[t]{2}{*}{ Época } & \multicolumn{3}{|c|}{ Média } & \multicolumn{3}{|c|}{ Variância } & \multicolumn{3}{|c|}{ QME } \\
\hline & OBS & FIL & WE & OBS & FIL & WE & FIL & WE & $\mathrm{F}$ \\
\hline & \multicolumn{9}{|c|}{ Eucalyptus grandis } \\
\hline CA5 & 15,0 & $18,6^{* *}$ & $15,4^{\mathrm{ns}}$ & 2,55 & $2,60^{\text {ns }}$ & $2,38^{\mathrm{ns}}$ & 12,83 & 0,73 & ** \\
\hline CA6 & 17,1 & $21,5^{* *}$ & $18,2^{\mathrm{ns}}$ & 2,54 & $2,42^{\mathrm{ns}}$ & $2,23^{\mathrm{ns}}$ & 19,99 & 3,23 & * \\
\hline CA7 & 17,5 & $24,7^{* *}$ & $20,6^{* *}$ & 2,34 & $2,49^{\mathrm{ns}}$ & $2,26^{\mathrm{ns}}$ & 21,93 & 9,76 & $* *$ \\
\hline CA8 & 15,4 & $24,3^{* *}$ & $19,7^{* *}$ & 2,63 & $2,59^{\mathrm{ns}}$ & $2,32^{\mathrm{ns}}$ & 78,54 & 21,75 & ns \\
\hline CA9 & 13,7 & $20,9^{* *}$ & $16,0^{* *}$ & 2,35 & $2,84^{\mathrm{ns}}$ & $2,48^{\mathrm{ns}}$ & 59,97 & 6,59 & $* *$ \\
\hline CV1 & 15,6 & $16,9^{*}$ & $14,4^{\mathrm{ns}}$ & 2,53 & $2,14^{\mathrm{ns}}$ & $2,01^{\mathrm{ns}}$ & 5,25 & 6,58 & ns \\
\hline \multirow[t]{2}{*}{ CV2 } & 16,9 & $27,5^{* *}$ & $21,9^{* *}$ & 2,35 & $2,44^{\mathrm{ns}}$ & $2,18^{\mathrm{ns}}$ & 111,10 & 25,32 & ns \\
\hline & \multicolumn{9}{|c|}{ Eucalyptus saligna } \\
\hline CA5 & 16,3 & $19,6^{* *}$ & $15,4^{\mathrm{ns}}$ & 2,82 & $2,62^{\text {ns }}$ & $2,38^{\mathrm{ns}}$ & 6,99 & 5,39 & ** \\
\hline CA6 & 17,1 & $19,5^{\mathrm{ns}}$ & $13,5^{\mathrm{ns}}$ & 2,71 & $2,16^{\mathrm{ns}}$ & $1,82^{\mathrm{ns}}$ & 12,48 & 26,15 & * \\
\hline CA7 & 18,7 & $23,2^{* *}$ & $16,0^{* *}$ & 2,08 & $2,47^{\text {ns }}$ & $2,02^{\mathrm{ns}}$ & 23,95 & 8,22 & $* *$ \\
\hline CA8 & 16,8 & $22,3^{\text {** }}$ & $14,9^{\text {ns }}$ & 2,61 & $2,52^{\mathrm{ns}}$ & $2,08^{\text {ns }}$ & 26,69 & 8,80 & ns \\
\hline CA9 & 13,0 & $25,0^{* *}$ & $17,2^{* *}$ & 2,29 & $3,13^{\mathrm{ns}}$ & $2,58^{\mathrm{ns}}$ & 165,20 & 19,71 & $* *$ \\
\hline CV1 & 15,9 & $16,2^{\text {ns }}$ & $11,6^{* *}$ & 2,60 & $1,92^{\text {ns }}$ & $1,69^{\mathrm{ns}}$ & 9,30 & 33,13 & $* *$ \\
\hline CV2 & 17,2 & $33,1^{* *}$ & $22.6^{* *}$ & 2,37 & $2,71^{\mathrm{ns}}$ & $2.25^{\mathrm{ns}}$ & 253,70 & 29,31 & ** \\
\hline
\end{tabular}

(1)OBS: valor observado; CA5: época 5 (20/10/2005); CA6: época 6 (21/11/2005); CA7: época 7 (13/12/2005); CA8: época 8 (14/2/2006); CA9: época 9 (3/4/2006); CV1: época 1 (3/10/2005); CV2: época $2(12 / 5 / 2006)$. ns Não-significativo. * e **Significativo a 5 e $1 \%$ pelo teste $t$ (compara a média observada com cada média estimada), pelo teste de Bartlett (compara a variância observada com cada variância estimada) e pelo teste F (compara o QME de um modelo com o QME do outro modelo). 
no experimento em casa de vegetação (CV1), onde o modelo de WE subestimou o NF. Nesta época CV1 ocorreram as maiores temperaturas do ar o que resultou em baixos valores de TAF estimado pelo modelo de WE em função do fato de que vários dias apresentaram $T \bar{m}$ maior que a Tot para esta espécie. Quanto ao valor de $\mathrm{Vp}$, que integra as estatísticas empíricas, o modelo de
WE foi superior (menor valor de $\mathrm{Vp}$ ) em relação ao modelo do Filocrono (maior valor de Vp), em todas as épocas, no campo e em casa de vegetação.

Quanto a E. saligna, a superioridade do modelo de WE não foi verificada na época 5 do experimento em campo (CA5) e na época 1 (CV1), do experimento em casa de vegetação, onde a maioria dos indicadores
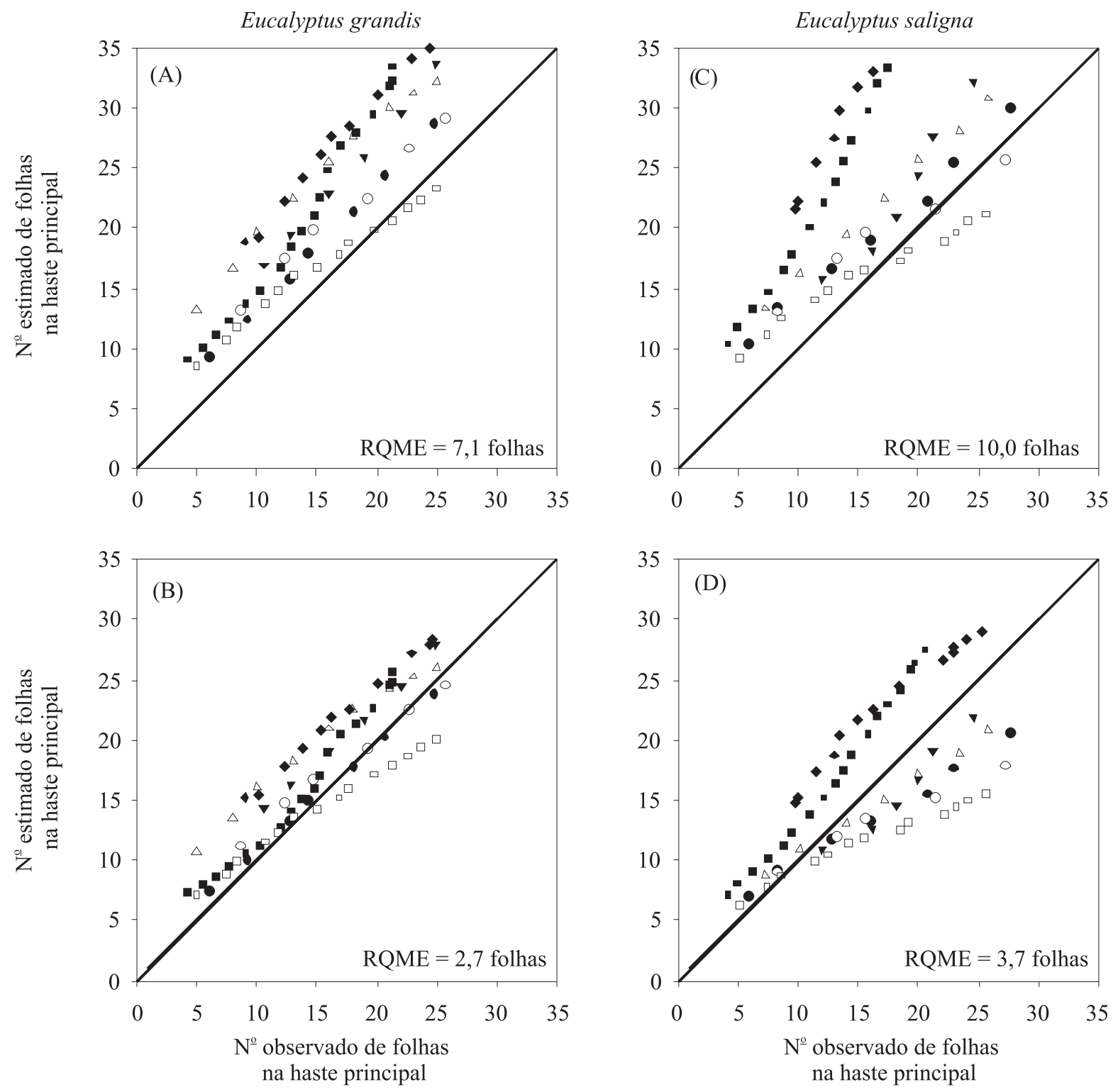

- CA5 $\circ$ CA6 $6 \mathrm{CA} 7 \triangle \mathrm{CA} 8 \bullet \mathrm{CA} 9 \square \mathrm{CV} 1 \bullet \mathrm{CV} 2$

Figura 1. Número de folhas na haste principal observado versus estimado pelo modelo do Filocrono (A, C) e pelo modelo de WE(B, D), nas duas espécies de eucalipto. A linha cheia transversal é a linha 1:1. CA5: época 5 (20/10/2005); CA6: época 6(21/11/2005); CA7: época 7(13/12/2005); CA8: época 8(14/2/2006); CA9: época 9(3/4/2006); CV1: época 1 (3/10/2005); CV2: época 2(12/5/2006). 
estatísticos apontaram melhor performance do modelo do Filocrono (Tabela 3). Nas outras épocas do experimento em campo (CA6, CA7, CA8 e CA9), e na segunda época do experimento em casa de vegetação (CV2), o modelo de WE foi superior ao modelo do Filocrono.

Deve ser dada preferência ao modelo de WE na estimativa do aparecimento de folhas em mudas de E. grandis e E. saligna. O modelo de WE também foi melhor na estimativa do aparecimento de folhas em espécies agrícolas anuais como cevada, sorgo e milho (Yan \& Hunt, 1999), trigo (Xue et al., 2004), melão (Streck et al., 2006) e batata (Streck et al., 2007) comparado com o modelo do Filocrono. A melhor performance do modelo de WE tem sido atribuída à resposta não-linear do aparecimento de folhas à temperatura usada no primeiro modelo (Xue et al., 2004; Streck et al., 2006, 2007). No modelo do Filocrono, a resposta do aparecimento de folhas à temperatura é linear, o que não é completamente aceita do ponto de vista biológico, pois próximo às temperaturas cardinais (temperatura base, ótima e máxima), a resposta da taxa de aparecimento de folhas à temperatura é não-linear (Streck, 2002; Xue et al., 2004). Em apenas uma pequena faixa entre a temperatura base e a temperatura ótima, a resposta da taxa de aparecimento de folhas à temperatura é linear (Streck, 2004), o que é contemplado na $\mathrm{f}(\mathrm{T})$ usada no modelo de WE.

Outra vantagem do modelo de WE é que os coeficientes do modelo têm significado biológico (Wang \& Engel, 1998) e definição operacional, como o coeficiente $\mathrm{TAF}_{\text {máx }}$ e os coeficientes da $\mathrm{f}(\mathrm{T})$, as temperaturas cardinais (base, ótima e máxima). É possível encontrar outras funções não-lineares para descrever a resposta do desenvolvimento à temperatura, mas algumas destas funções têm a desvantagem de ser composta por coeficientes empíricos, que não apresentam significado biológico, como a função exponencial dupla (Streck, 2004), as quais devem ser evitadas. O modelo

Tabela 3. Valores das estatísticas empíricas usadas na avaliação dos modelos do Filocrono (FIL) e Wang e Engel (WE) para estimativa do aparecimento de folhas em duas espécies de eucalipto, nas diferentes épocas de semeadura em campo (CA5, CA6, CA7, CA8, CA9) e em casa de vegetação (CV1 e CV2) ${ }^{(1)}$.

\begin{tabular}{|c|c|c|c|c|c|c|c|c|c|c|c|c|c|c|}
\hline \multirow[t]{2}{*}{ Estatísticas } & \multicolumn{2}{|c|}{ CA5 } & \multicolumn{2}{|c|}{ CA6 } & \multicolumn{2}{|c|}{ CA7 } & \multicolumn{2}{|c|}{ CA8 } & \multicolumn{2}{|c|}{ CA9 } & \multicolumn{2}{|c|}{ CV1 } & \multicolumn{2}{|c|}{ CV2 } \\
\hline & FIL & WE & FIL & WE & FIL & WE & FIL & WE & FIL & WE & FIL & WE & FIL & WE \\
\hline & \multicolumn{14}{|c|}{ Eucalyptus grandis } \\
\hline $\mathrm{dw}$ & $\begin{array}{c}0,922 \\
\text { (2) }\end{array}$ & $\begin{array}{c}0,994 \\
(1)\end{array}$ & $\begin{array}{c}0,869 \\
(2)\end{array}$ & $\begin{array}{c}0,971 \\
(1)\end{array}$ & $\begin{array}{c}0,706 \\
\text { (2) }\end{array}$ & $\begin{array}{c}0,906 \\
(1)\end{array}$ & $\begin{array}{c}0,490 \\
(2)\end{array}$ & $\begin{array}{c}0,868 \\
\text { (1) }\end{array}$ & $\begin{array}{c}0,744 \\
(2)\end{array}$ & $\begin{array}{c}0,951 \\
(1)\end{array}$ & $\begin{array}{c}0,936 \\
\text { (2) }\end{array}$ & $\begin{array}{c}0,953 \\
(1)\end{array}$ & $\begin{array}{c}0,698 \\
\text { (2) }\end{array}$ & $\begin{array}{c}0,911 \\
\text { (1) }\end{array}$ \\
\hline $\mathrm{r}$ & $\begin{array}{c}0,998 \\
(2)\end{array}$ & $\begin{array}{c}0,999 \\
(1)\end{array}$ & $\begin{array}{c}0,995 \\
(2)\end{array}$ & $\begin{array}{c}0,997 \\
\text { (1) }\end{array}$ & $\begin{array}{c}0,998 \\
\text { (2) }\end{array}$ & $\begin{array}{c}0,999 \\
(1)\end{array}$ & $\begin{array}{c}0,997 \\
\text { (1) }\end{array}$ & $\begin{array}{c}0,991 \\
(2)\end{array}$ & $\begin{array}{c}0,991 \\
(1)\end{array}$ & $\begin{array}{c}0,989 \\
(2)\end{array}$ & $\begin{array}{c}0,995 \\
(2)\end{array}$ & $\begin{array}{c}0,997 \\
\text { (1) }\end{array}$ & $\begin{array}{c}0,997 \\
\text { (2) }\end{array}$ & $\begin{array}{c}0,998 \\
\text { (1) }\end{array}$ \\
\hline c & $\begin{array}{c}0,922 \\
(2)\end{array}$ & $\begin{array}{c}0,994 \\
\text { (1) }\end{array}$ & $\begin{array}{c}0,864 \\
\text { (2) }\end{array}$ & $\begin{array}{c}0,968 \\
\text { (1) }\end{array}$ & $\begin{array}{c}0,705 \\
\text { (2) }\end{array}$ & $\begin{array}{c}0,905 \\
\text { (1) }\end{array}$ & $\begin{array}{c}0,489 \\
\text { (2) }\end{array}$ & $\begin{array}{c}0,860 \\
\text { (1) }\end{array}$ & $\begin{array}{c}0,737 \\
\text { (2) }\end{array}$ & $\begin{array}{c}0,941 \\
\text { (1) }\end{array}$ & $\begin{array}{c}0,949 \\
\text { (1) }\end{array}$ & $\begin{array}{c}0,933 \\
\text { (2) }\end{array}$ & $\begin{array}{c}0,696 \\
\text { (2) }\end{array}$ & $\begin{array}{c}0,907 \\
\text { (1) }\end{array}$ \\
\hline RQME & $\begin{array}{c}3,582 \\
\text { (2) }\end{array}$ & $\begin{array}{c}0,857 \\
\text { (1) }\end{array}$ & $\begin{array}{c}4,642 \\
\text { (2) }\end{array}$ & $\begin{array}{c}4,270 \\
\text { (1) }\end{array}$ & $\begin{array}{c}4,972 \\
\text { (2) }\end{array}$ & $\begin{array}{c}4,543 \\
\text { (1) }\end{array}$ & $\begin{array}{c}4,708 \\
\text { (2) }\end{array}$ & $\begin{array}{c}4,441 \\
\text { (1) }\end{array}$ & $\begin{array}{c}4,573 \\
\text { (2) }\end{array}$ & $\begin{array}{c}4,006 \\
\text { (1) }\end{array}$ & $\begin{array}{c}4,145 \\
\text { (2) }\end{array}$ & $\begin{array}{c}3,800 \\
\text { (1) }\end{array}$ & $\begin{array}{c}5,668 \\
\text { (2) }\end{array}$ & $\begin{array}{c}5,070 \\
\text { (1) }\end{array}$ \\
\hline BIAS & $\begin{array}{c}0,237 \\
\text { (2) }\end{array}$ & $\begin{array}{c}0,024 \\
\text { (1) }\end{array}$ & $\begin{array}{c}0,256 \\
\text { (2) }\end{array}$ & $\begin{array}{c}0,063 \\
\text { (1) }\end{array}$ & $\begin{array}{c}0,409 \\
\text { (2) }\end{array}$ & $\begin{array}{c}0,177 \\
\text { (1) }\end{array}$ & $\begin{array}{c}0,572 \\
(2)\end{array}$ & $\begin{array}{c}0,277 \\
\text { (1) }\end{array}$ & $\begin{array}{c}0,527 \\
\text { (2) }\end{array}$ & $\begin{array}{c}0,171 \\
(1)\end{array}$ & $\begin{array}{c}0,087 \\
\text { (2) }\end{array}$ & $\begin{array}{c}-0,073 \\
\text { (1) }\end{array}$ & $\begin{array}{c}0,474 \\
\text { (2) }\end{array}$ & $\begin{array}{c}0,180 \\
\text { (1) }\end{array}$ \\
\hline $\mathrm{Vp}$ & 10 & 5 & 10 & 5 & 10 & 5 & 9 & 6 & 9 & & 9 & & 1 & 5 \\
\hline & \multicolumn{14}{|c|}{ Eucalyptus saligna } \\
\hline $\mathrm{dw}$ & $\begin{array}{c}0,936 \\
\text { (1) }\end{array}$ & $\begin{array}{c}0,890 \\
(2)\end{array}$ & $\begin{array}{c}0,900 \\
\text { (1) }\end{array}$ & $\begin{array}{c}0,763 \\
\text { (2) }\end{array}$ & $\begin{array}{c}0,783 \\
\text { (1) }\end{array}$ & $\begin{array}{c}0,878 \\
(2)\end{array}$ & $\begin{array}{c}0,838 \\
(2)\end{array}$ & $\begin{array}{c}0,920 \\
\text { (1) }\end{array}$ & $\begin{array}{c}0,557 \\
(2)\end{array}$ & $\begin{array}{c}0,871 \\
(1)\end{array}$ & $\begin{array}{c}0,907 \\
\text { (1) }\end{array}$ & $\begin{array}{c}0,722 \\
\text { (2) }\end{array}$ & $\begin{array}{c}0,540 \\
\text { (2) }\end{array}$ & $\begin{array}{c}0,877 \\
\text { (1) }\end{array}$ \\
\hline $\mathrm{r}$ & $\begin{array}{c}0,996 \\
\text { (2) }\end{array}$ & $\begin{array}{c}0,997 \\
\text { (1) }\end{array}$ & $\begin{array}{c}0,986 \\
\text { (2) }\end{array}$ & $\begin{array}{c}0,993 \\
\text { (1) }\end{array}$ & $\begin{array}{c}0,972 \\
\text { (2) }\end{array}$ & $\begin{array}{c}0,974 \\
\text { (1) }\end{array}$ & $\begin{array}{c}0,998 \\
\text { (2) }\end{array}$ & $\begin{array}{c}0,999 \\
\text { (1) }\end{array}$ & $\begin{array}{c}0,996 \\
\text { (1) }\end{array}$ & $\begin{array}{c}0,995 \\
\text { (2) }\end{array}$ & $\begin{array}{c}0,989 \\
(2)\end{array}$ & $\begin{array}{c}0,992 \\
\text { (1) }\end{array}$ & $\begin{array}{c}0,989 \\
\text { (1) }\end{array}$ & $\begin{array}{c}0,988 \\
\text { (2) }\end{array}$ \\
\hline c & $\begin{array}{c}0,932 \\
(1)\end{array}$ & $\begin{array}{c}0,887 \\
(2)\end{array}$ & $\begin{array}{c}0,887 \\
\text { (1) }\end{array}$ & $\begin{array}{c}0,758 \\
\text { (2) }\end{array}$ & $\begin{array}{c}0,761 \\
(2)\end{array}$ & $\begin{array}{c}0,855 \\
\text { (1) }\end{array}$ & $\begin{array}{c}0,835 \\
(2)\end{array}$ & $\begin{array}{c}0,919 \\
\text { (1) }\end{array}$ & $\begin{array}{c}0,563 \\
(2)\end{array}$ & $\begin{array}{c}0,866 \\
\text { (1) }\end{array}$ & $\begin{array}{c}0,897 \\
\text { (1) }\end{array}$ & $\begin{array}{c}0,716 \\
(2)\end{array}$ & $\begin{array}{c}0,534 \\
(2)\end{array}$ & $\begin{array}{c}0,867 \\
\text { (1) }\end{array}$ \\
\hline RQME & $\begin{array}{c}3,573 \\
\text { (1) }\end{array}$ & $\begin{array}{c}4,099 \\
\text { (2) }\end{array}$ & $\begin{array}{c}4,419 \\
\text { (2) }\end{array}$ & $\begin{array}{c}3,681 \\
\text { (1) }\end{array}$ & $\begin{array}{c}4,816 \\
\text { (2) }\end{array}$ & $\begin{array}{c}3,998 \\
\text { (1) }\end{array}$ & $\begin{array}{c}4,719 \\
\text { (2) }\end{array}$ & $\begin{array}{c}3,865 \\
\text { (1) }\end{array}$ & $\begin{array}{c}5,003 \\
(2)\end{array}$ & $\begin{array}{c}4,143 \\
\text { (1) }\end{array}$ & $\begin{array}{c}4,021 \\
\text { (2) }\end{array}$ & $\begin{array}{c}3,407 \\
\text { (1) }\end{array}$ & $\begin{array}{c}6,055 \\
\text { (2) }\end{array}$ & $\begin{array}{c}5,004 \\
\text { (1) }\end{array}$ \\
\hline BIAS & $\begin{array}{c}0,207 \\
\text { (2) }\end{array}$ & $\begin{array}{c}-0,162 \\
\text { (1) }\end{array}$ & $\begin{array}{c}0,209 \\
\text { (2) }\end{array}$ & $\begin{array}{c}0,141 \\
\text { (1) }\end{array}$ & $\begin{array}{c}0,202 \\
\text { (2) }\end{array}$ & $\begin{array}{c}-0,145 \\
\text { (1) }\end{array}$ & $\begin{array}{c}0,322 \\
\text { (2) }\end{array}$ & $\begin{array}{c}-0,106 \\
\text { (1) }\end{array}$ & $\begin{array}{c}0,928 \\
\text { (2) }\end{array}$ & $\begin{array}{c}0,321 \\
\text { (1) }\end{array}$ & $\begin{array}{c}0,015 \\
\text { (1) }\end{array}$ & $\begin{array}{c}-0,272 \\
\text { (2) }\end{array}$ & $\begin{array}{c}0,786 \\
\text { (2) }\end{array}$ & $\begin{array}{c}0,219 \\
\text { (1) }\end{array}$ \\
\hline Vp & 7 & 8 & 8 & 7 & 9 & 6 & 10 & 5 & 9 & 6 & 7 & 8 & 9 & 6 \\
\hline
\end{tabular}

${ }^{(1)}$ Valores entre parênteses, abaixo de cada estatística, são atribuídos ao cálculo da estatística Vp (valor 1 - melhor modelo em cada indicador estatístico; valor 2 - pior modelo em cada indicador estatístico); dw: índice de concordância; r: coeficiente de correlação; c: índice c; RQME: raiz quadrada do quadrado médio do erro; BIAS: índice BIAS; Vp: escores estatísticos ponderados; CA5: época 5 (20/10/2005); CA6: época 6 (21/11/2005); CA7: época 7 (13/12/2005); CA8: época 8 (14/2/2006); CA9: época 9 (3/4/2006); CV1: época 1 (3/10/2005); CV2: época 2 (12/5/2006). 
de WE também tem a vantagem de combinar os efeitos do ambiente sobre a TAF de forma multiplicativa, o que é realístico biologicamente, uma vez que as interações entre ambiente e o desenvolvimento vegetal são geralmente multiplicativas (Streck et al., 2003).

\section{Conclusões}

1. O modelo de Wang e Engel é melhor do que o modelo do Filocrono em estimar o número de folhas na haste principal em mudas de Eucalyptus grandis e E. saligna cultivadas em épocas diferentes em experimentos em campo e casa de vegetação.

2. A resposta não-linear da taxa de aparecimento de folhas em função da temperatura assumida no modelo de Wang e Engel é válida para E. grandis e E. saligna.

\section{Agradecimentos}

Ao CNPq, pelas bolsas concedidas; a Joel Cordeiro da Silva e Lindolfo Storck, pelo auxílio na análise estatística dos dados; aos acadêmicos do curso de Engenharia Florestal da UFSM, Felipe Susin, Magnos Alan Vivian, Márcio Carlos Navroski e Weslley Wilker Moraes, pelo auxílio na coleta dos dados.

\section{Referências}

ALMEIDA, A.C.; LANDSBERG, J.J.; SANDS, P.J. Parameterisation of 3-PG model for fast-growing Eucalyptus grandis plantations. Forest Ecology and Management, v.193, p.179-195, 2004.

ASSOCIAÇÃO BRASILEIRA DE FLORESTAS PLANTADAS. Anuário Estatístico da ABRAF: ano base 2005, Brasília, 2006. Disponível em: < http://www.ipef.br/estatisticas/relatorios/anuarioABRAF-2006.pdf>. Acesso em: 7 dez. 2006.

CAMARGO, A.P. de; SENTELHAS, P.C. Avaliação do desempenho de diferentes métodos de estimativa da evapotranspiração potencial no estado de São Paulo, Brasil. Revista Brasileira de Agrometeorologia, v.5, p.89-97, 1997.

CARNEIRO, J.G. de A. Produção e controle de qualidade de mudas florestais. Curitiba: UFPR/FUPEF/UNEF, 1995. 451p.

EMBRAPA. Centro Nacional de Pesquisa de Solos. Sistema brasileiro de classificação de solos. Brasília: Embrapa-SPI; EmbrapaCNPS, 1999. 412p.

FRANK, A.B.; BAUER, A. Phyllochron differences in wheat, barley and forage grasses. Crop Science, v.35, p.19-23, 1995.

HODGES, T.F. Predicting crop phenology. Boca Raton: CRC, 1991, $233 p$.

KALLARACKAL, J.; SOMER, C.K. An ecophysiological evaluation of the suitability of Eucalyptus grandis for planting in the tropics. Forest Ecology and Management, v.95, p.53-61, 1997.
KLEPPER, B.; RICKMAN, R.W.; PETERSON, C.M. Quantitative characterization of vegetative development in small cereal grains. Agronomy Journal, v.74, p.789-792, 1982.

LEITE, H.G.; ANDRADE, V.C.L. de. Um método para condução de inventários florestais sem o uso de equações volumétricas. Revista Árvore, v.26, p.321-328, 2002.

MARTINS, F.B. Desenvolvimento e estresse hídrico em mudas de Eucalyptus grandis (Hill ex Maiden) e Eucalyptus saligna (Smith). 2007. 57p. Dissertação (Mestrado) - Universidade Federal de Santa Maria, Santa Maria.

MATTHEWS, R.B.; HUNT, L.A. GUMCAS: a model describing the growth of cassava (Manihot esculenta (L.) Crantz). Field Crops Reseach, v.39, p.69-84, 1994.

MITCHELL, P.L. Misuse of regression for empirical validation of models. Agricultural Systems, v.54, p.313-326, 1997.

NIETO, V.M.; RODRIGUEZ, J. Eucalyptus saligna Sm. In: VOZZO, J.A. (Ed.). Tropical Tree Seed Manual. Washington DC: USDA Forest Service, 2003. p.468-469.

SANDS, P.J.; LANDSBERG, J.J. Parameterisation of 3-PG for plantation grown Eucalyptus globulus. Forest Ecology and Management, v.163, p.273-292, 2002.

SANTOS, P.E.T.; GERALDI, I.O.; GARCIA, J.N. Estimativas de parâmetros genéticos de propriedades físicas e mecânicas da madeira de Eucalyptus grandis. Scientia Forestalis, v.63, p.54-64, 2003.

SCHNEIDER, P.R. Análise de regressão aplicada à engenharia florestal. 2.ed. Santa Maria: Ed. UFSM/CEPEF, 1998. 236p.

SCURFIELD, G. The effects of temperature and day length on species of Eucalyptus. Australian Journal of Botany, v.9, p.37-56, 1961. SOCIEDADE BRASILEIRA DE CIÊNCIA DO SOLO - SBCS. Comissão de Química e Fertilidade do Solo. Manual de adubação e calagem para os estados do Rio Grande do Sul e de Santa Catarina. 10.ed. Porto Alegre: SBCS/CQFS, 2004. 394p.

STAPE, J.L.; BINKLEY, D.; RYAN, M.G. Eucalyptus production and the supply, use and efficiency of use of water, light and nitrogen across a geographic gradient in Brazil. Forest Ecology and Management, v.193, p.17-31, 2004.

STRECK, N.A. A generalized nonlinear air temperature response function for node appearance rate in muskmelon (Cucumis melo L.). Revista Brasileira de Agrometeorologia, v.10, p.105-111, 2002.

STRECK, N.A. A temperature response function for development of the crysanthemum (Chrysanthemum $x$ morifolium Ramat.). Ciência Rural, v.34, p.49-54, 2004.

STRECK, N.A.; LAGO, I.; BURIOL, G.A.; HELDWEIN, A.B.; TIBOLA, T. A non-linear model to simulate node appearance in muskmelon (Cucumis melo L.) grown inside plastic greenhouse as a function of air temperature. Revista Brasileira de Agrometeorologia, v.14, p.210-216, 2006.

STRECK, N.A.; LAGO, I.; PAULA, F.L.M.; BISOGNIN, D.A.; HELDWEIN, A.B. Improving predictions of leaf appearance in field grown potato. Scientia Agricola, v.64, p.12-18, 2007.

STRECK, N.A.; WEISS, A.; XUE, Q.; BAENZIGER, P.S. Incorporating a chronology response function into the prediction of leaf appearance rate in winter wheat. Annals of Botany, v.92, p.181190, 2003. 
THIERSCH, A. Eficiência das distribuições diamétricas para prognose da produção de Eucalyptus camaldulensis. 1997. 155p. Dissertação (Mestrado) - Universidade Federal de Lavras, Lavras.

WANG, E.; ENGEL, T. Simulation of phenological development of wheat crops. Agricultural Systems, v.58, p.1-24, 1998.

WILlmotT, C.J. On the validation models. Physical Geography, v.2, p.184-194, 1981.
XUE, Q.; WEISS, A.; BAENZIGER, P.S. Predicting leaf appearance in field-grown winter wheat: evaluating linear and non-linear models. Ecological Modelling, v.175, p.261-270, 2004.

YAN, W.; HUNT, L.A. An equation for modelling the temperature response of plants using only the cardinal temperatures. Annals of Botany, v.84, p.607-614, 1999.

Recebido em 19 de março de 2007 e aprovado em 29 de junho de 2007 